\section{COPD: Nicht alle Patienten brauchen Dreifachtherapie}

Chapman KR et al. Long-term Triple Therapy Deescalation to Indacaterol/Glycopyrronium in COPD Patients (SUNSET): a Randomized, Double-Blind, Triple-Dummy Clinical Trial. AJRCCM 2018; DOI 10.1164/rccm.201803-0405OC

Obwohl das aktuelle GOLD Statement eine Dreifachkombination aus LAMA, LABA und inhalativem Kortikosteroid nur bei Hochrisikopatienten empfiehlt, werden die meisten COPD-Patienten nach diesem Konzept behandelt. Chapman und Team haben nun erstmal mit einer randomisierten kontrollierten Studie getestet, ob sich ein abruptes Absetzen des Steroids bei Betroffenen mit seltenen Exazerbationen negativ auf Lungenfunktion und Exazerbationsrate auswirkt.

Ein Großteil der COPD-Patienten wird heute auch bei niedrigem Exazerbationsrisiko mit der Dreifachkombination aus LAMA, LABA und inhalativem Kortikosteroid behandelt. Da insbesondere letzteres die Gefahr von langfristigen Nebenwirkungen mit sich bringt, stellt sich die Frage nach den Konsequenzen einer Deeskalationen bei Patienten, bei denen es zuletzt selten oder gar nicht zu Krankheitsverschlimmerungen gekommen ist. Chapman und Kollegen haben daher eine randomisierte doppelblinde kontrollierte klinische Studie über 26 Wochen durchgeführt, um die Wirksamkeit und Sicherheit einer direkten Deeskalation der Dreifachtherapie zu einer Zweifachtherapie mit LAMA und LABA zu testen.

Geeignete Probanden mussten folgende Kriterien erfüllen:

- Alter ab 40 Jahren,

- stabile COPD,

- $\mathrm{FEV}_{1}$ zwischen $40 \%$ und $80 \%$ vom Sollwert,

- Nikotinabusus von mindestens 10 packyears,

- maximal 1 mittlere bis schwere Exazerbation im vergangenen Jahr.
Patienten mit einem Wert von über 600 Eosinophilen/ul durften nicht an der Studie teilnehmen. Alle Patienten erhielten zunächst die Dreifachtherapie. Im Anschluss bildeten die Forscher zwei Studiengruppen:

- Gruppe 1: Deeskalation auf eine Zweifachtherapie mit Indacaterol/ Glycopyrronium $110 / 50 \mu \mathrm{g} 1 \times$ täglich.

- Gruppe 2: Fortführung der Dreifachtherapie mit Tiotropium $18 \mu \mathrm{g}$ $1 \times$ täglich und Salmeterol/Fluticason $50 / 500 \mu \mathrm{g} 2 \times$ täglich.

Als primärer klinischer Endpunkt diente die $\mathrm{FEV}_{1}$ als Standardmarker für die Lungenfunktion. Sekundärer Endpunkt war die Rate moderater bis schwerer Exazerbationen. Um eine Subgruppenanalyse durchführen zu können, registrierten Chapman und sein Team von jedem Probanden die entsprechenden Eosinophilenwerte. Jeder Verdacht auf eine Arzneimittelnebenwirkung wurde aufgezeichnet.

\section{Ergebnisse}

928 der zunächst 1053 randomisierten Patienten beendeten die Studie, 456 in Gruppe 1 und 472 in Gruppe 2. Sie waren durchschnittlich 65,3 Jahre alt (Standardabweichung 7,8), 70,6\% von ihnen männlich. Die FEV ${ }_{1}$ nach Inhalation eines Brochodilatators lag vor Studienbeginn im Mittel bei 56,6\% vom Sollwert (9,97\%), 34,1\% aller Patienten hatten im vergangenen Jahr eine Exazerbation, 23,2\% zeigten Eosinophilenzahlen über $300 / \mu l$.

Nach der Deeskalation in Gruppe 1 verschlechterte sich die Lungenfunktion nachweisbar, sodass die Forscher eine Nicht-Unterlegenheit der Zweifachtherapie nicht belegen konnten. So lag die Abnahme bei $-26 \mathrm{~mL}$ mit einem $95 \%$ Vertrauensintervall von -53 bis $1 \mathrm{~mL}$, was über der vorab festgelegten Grenze von $-50 \mathrm{~mL}$ lag.
Was den sekundären Endpunkt - die Exazerbationsrate - betrifft, hatte die Deeskalation dagegen in der Gesamtanalyse keine negativen Veränderungen zur Folge. In der Subgruppenanalyse zeigten jedoch Patienten mit Eosinophilenzahlen über $300 / \mu l$ ein höheres Risiko für akute Krankheitsverschlechterungen und schienen daher im direkten Vergleich besonders von einer Fortführung der Dreifachtherapie zu profitieren.

FAZIT

COPD-Patienten mit niedrigem Risiko für Exazerbationen zeigten bei direkter Deeskalation der Dreifachtherapie mit inhalativem Kortikosteroid eine minimale Reduktion der Lungenfunktion bei gleichbleibender Exazerbationsrate. Da allerdings für Patienten mit Eosinophilenzahlen über $300 / \mu \mathrm{l}$ das Exazerbationsrisiko anstieg, empfehlen die Autorinnen/Autoren eine stärkere Personalisierung der medikamentösen Therapie.

Dipl.-Psych. Annika Simon, Hannover 